\title{
Single umbilical artery: What does it mean for the fetus? A case-control analysis of pathologically ascertained cases
}

Sandra Prucka, $M S^{1}$, Michele Clemens, $M S^{1,2}$, Catherine Craven, $\dagger M D^{3}$, and Elizabeth McPherson, $M D^{4}$

\begin{abstract}
Purpose: To ascertain the frequency of chromosomal and other anomalies in fetuses with single umbilical artery. Methods: Placentas with single umbilical artery were identified from hospital pathology laboratory records. For each identified case, the next consecutive placenta with two umbilical arteries served as a control. Pathology records, maternal histories, and prenatal ultrasounds when available were reviewed for congenital anomalies, pregnancy complications, and maternal characteristics. When indicated, placental specimens, amniocytes, or neonatal bloods were karyotyped. Results: Single umbilical artery existed in 2.0\% (97/4846) of pathological specimens. Fetuses with single umbilical artery had significantly more chromosomal $(10.3 \%$ vs. $1.0 \%)$ and other congenital anomalies (27\% vs. $8 \%)$. Conclusions: The high incidence of major chromosomal and congenital anomalies justifies detailed fetal ultrasonography, echocardiography, and amniocentesis for karyotype when single umbilical artery is discovered during routine ultrasound. Genet Med 2004:6(1):54-57.
\end{abstract}

Key Words: single umbilical artery, chromosome anomalies, detailed ultrasound, karyotype, fetal echocardiography

The umbilical cord forms between 13 and 38 days after conception and normally serves as the conduit for two umbilical arteries and one umbilical vein.1,2 In some cases only a single umbilical artery (SUA) is present. Some report that this umbilical abnormality can be observed as early as 13 weeks. However, it is typically diagnosed in the third trimester. ${ }^{1,3}$ There are three theories to explain how a SUA may form during development. ${ }^{1,4}$ The first is that a primary agenesis of one umbilical artery results in a SUA. Another theory attributes the phenomenon to a secondary atrophy or atresia of a previously normal umbilical artery. A third theory describes a persistence of the original allantoic artery of the body stalk as an explanation for SUA. Embryological considerations, as well as the detection of occluded remnants of a second umbilical artery in some SUA fetuses, suggest that the second theory is the most likely explanation..$^{1,4,5}$

In a review of eight studies of SUA in the literature, Persutte and Hobbins ${ }^{1}$ reported an incidence of SUA in 1.5\% in spontaneous abortuses, $7 \%$ of pregnancies that were terminated because of a serious malformation, $0.2 \%$ to $1.6 \%$ of euploid

\footnotetext{
From the ${ }^{I}$ Department of Human Genetics, University of Pittsburgh Graduate School of Public Health, Pittsburgh Pennsylvania; Departments of ${ }^{2}$ Genetics and ${ }^{3}$ Pathology, MageeWomen's Hospital, Pittsburgh, Pennsylvania; and ${ }^{4}$ Medical Genetics Services, Marshfield Clinic, Marshfield, Wisconsin.

$\dagger$ Deceased.

Dr. Elizabeth McPherson, Medical Genetics Services, Marshfield Clinic, 1000 North Oak Avenue 1A4, Marshfield, WI 54449.

Received: July 30, 2003.

Accepted: September 23, 2003.
}

DOI: 10.1097/01.GIM.0000105743.91723.B0 fetuses who underwent prenatal ultrasound examination, $9 \%$ to $11 \%$ of aneuploid fetuses, and $0.5 \%$ to $2.5 \%$ of uncomplicated neonates. The reported incidence of SUA varies depending on the method used to identify its occurrence, being highest in abortuses and autopsies and relatively low on ultrasound and in term neonates. ${ }^{1,6-8}$ Although there has been no evidence supporting any genetic etiology or familial tendency of this condition, it is known that SUA occurs more frequently in twin births $(4.6 \%)$ versus singletons $(1 \%))_{.}^{4,9}$

The risk for congenital anomalies in infants with SUA also depends on the method of ascertainment, being highest at autopsy, but cases diagnosed at ultrasound or at term delivery still have an increased risk of anomalies compared to infants with two umbilical arteries. In the metaanalysis of Thummala et al., ${ }^{8}$ nine autopsy-based studies show incidences of anomalies ranging from $25 \%$ to $81.8 \%$ with a mean of $61.4 \%$, whereas 23 studies based on screening of placentas or umbilical stumps of term neonates show anomalies ranging from $8.7 \%$ to $66.7 \%$ with a mean of $27 \%$. Observed anomalies most often involve the urogenital, gastrointestinal cardiovascular, respiratory, and central nervous systems, as well as the face. ${ }^{9}$ Similar anomalies have been noted on ultrasound among cases ascertained prenatally. ${ }^{1,3}$ We decided to use pathology as the means of ascertainment because we felt it was more accurate than ultrasound, despite recent advances with color imaging. Also we wanted data applicable to all stages of pregnancy, including the second trimester when decisions about amniocentesis and pregnancy intervention are being made. Therefore, we decided to include all cases of pathologically confirmed SUA regardless of gestational age and infant condition at delivery. We retrospectively reviewed the medical records, laboratory results, 
and ultrasound of 2 years of SUA placental specimens at a large high-risk maternity center.

\section{PATIENTS AND METHODS}

Placentas with a SUA, as well as control placentas with a 3-vessel cord, were obtained through examination of pathology records from the Pathology Department at Magee-Women's Hospital in Pittsburgh, Pennsylvania during a 2-year period from 1998 to 2000. During the period of the study, there were 21,861 deliveries at the institution of which 4,846 had placentas submitted to pathology. In all second and third trimester placentas and products of conception, the umbilical cord was examined as part of routine evaluation. All placentas with two vessel cords identified during the study period were included as cases, and for each, the next consecutive placenta with a 3-vessel cord served as the control. The women in the study, as well as their fetuses, were selected regardless of age, ethnicity, or socioeconomic status. Pathology records were reviewed to compare information on maternal complications, twinning, gestational age, birth weight, maternal age, and congenital anomalies. Charts of the mothers were used as needed to verify data from the pathology report. In total, there were 97 cases and 97 controls analyzed in this study. The pregnancy outcomes of cases and controls were recorded, and the number of anomalies in each category was tabulated. Not all women in the case and control populations had an ultrasound performed during their pregnancy. However, all available ultrasound data were reviewed. When clinically indicated, placental samples, amniocytes, or neonatal blood specimens were analyzed for chromosomal abnormalities through standard karyotyping procedures.

\section{RESULTS}

SUA occurred in $2.0 \%$ (97/4846) of the placentas submitted to pathology over this 2-year period.

Various chromosomal anomalies were observed in both the SUA and control groups (Table 1). In the SUA group, chromosomal anomalies included 45,X (3), trisomy 13 (3), trisomy 18 (2), triploidy (1), and balanced de novo t $(9 ; 17)$ (1). Congenital anomalies observed in this group included FAVS (Facioau-

Table 1

Chromosomal anomalies in cases and controls

\begin{tabular}{lcccc}
\hline Cases & & & \multicolumn{2}{c}{ Controls } \\
\cline { 1 - 2 } Chromosomal anomaly & Observed & & Chromosomal anomaly & Observed \\
\hline $45, \mathrm{X}$ & 3 & & $92, \mathrm{XXYY} / 46, \mathrm{XY}$ & 1 \\
Trisomy 13 & 3 & & & \\
Trisomy 18 & 2 & & \\
Triploidy & 1 & & 1 \\
Balanced $\mathrm{t}(9 ; 17)$ & 1 & & \\
Total & 10 & &
\end{tabular}

riculovertebral spectrum) (4), congenital heart disease (4), VACTERL (Vertebral-anal-cardiac-tracheoesophageal fistularenal-limb) (3), sirenomelia (2), anencephaly (2), limb-body wall defect (2), cleft lip/palate (2), hydrocephalus (1), cardiac rhabdomyoma (1), acardiac twin (1), renal (1), heterotaxy (1), craniocerebellar-cardiac syndrome (1), and isolated short femur (with no craniofacial anomalies) (1).

In the control group, only one chromosome anomaly, a 92,XXYY/46,XY mosaic liveborn with developmental delay and minor dysmorphic features, was found. Congenital anomalies observed in this group included renal (2), hydrocephalus (2), thanatophoric dysplasia (1), gastroschisis (1), sacral agenesis (1), and hypospadias (1).

A significant increase in combined chromosomal and congenital anomalies occurred in cases versus controls, with $37.1 \%$ of the cases displaying major anomalies compared with $9.3 \%$ of controls. Looking specifically at chromosomal anomalies, we saw 10/97 (10.31\%) of the SUAs with chromosomal anomalies versus $1 / 97(1.03 \%)$ of the controls with chromosomal abnormalities.

The maternal ages in cases and controls of our study population reflected the ages of patients in our high-risk maternity setting, with a mean age of 29.06 years in cases and 29.30 years in controls. Looking at the maternal ages of our study population revealed that $26(26.80 \%)$ of the cases and $26(26.80 \%)$ of the controls were 35 years of age or older. Of the 10 cases observed to have a chromosomal condition, only $2(20 \%)$ were over 35 years of age (Table 1 ).

Small for gestational age (SGA) was defined as below the $3 \mathrm{rd}$ percentile and large for gestational age (LGA) as above the 97 th percentile on the charts of Usher and McLean. ${ }^{10}$ Using these criteria those born after 24 weeks, whose weights were available, were analyzed. It was determined that $11 / 70$ cases $(15.71 \%)$ and $12 / 80$ controls $(15.00 \%)$ were LGA, whereas $16 / 70$ cases $(22.86 \%)$ and $7 / 80$ controls $(8.75 \%)$ were SGA (Table 2). Twinning was observed in $8 \%$ of cases in comparison to $5 \%$ of controls, whereas maternal nongestational diabetes was seen in $8 \%$ of cases and $4 \%$ of controls.

Ultrasound information was available on 62 of the SUA mothers, of which 38 (61.3\%) ultrasounds detected the SUA. Of the 36 SUA cases with anomalies, prenatal ultrasound data are only available on 31 of the pregnancies. The five anomalies where no ultrasound data were available include one case of triploidy, one case of balanced $t(9 ; 17)$, one case of bilateral cleft

Table 2

Size for gestational age in cases and controls

\begin{tabular}{lccc}
\hline Size for gestational age & $\begin{array}{c}\text { Cases } \\
(\mathrm{n}=70)\end{array}$ & $\begin{array}{c}\text { Controls } \\
(\mathrm{n}=80)\end{array}$ & $t$ test \\
\hline $\mathrm{LGA}^{a}$ & $11(15.71 \%)$ & $12(15.00 \%)$ & $\mathrm{P}=0.5786$ \\
$\mathrm{AGA}^{b}$ & $43(61.43 \%)$ & $61(76.25 \%)$ & $\mathrm{P}=0.1015$ \\
$\mathrm{SGA}^{c}$ & $16(22.86 \%)$ & $7(8.75 \%)$ & $\mathrm{P}=0.0013^{*}$ \\
\hline
\end{tabular}

${ }^{a}$ Large for gestational age; ${ }^{b}$ appropriate for gestational age; ${ }^{c}$ small for gestational age.

${ }^{\star}$ Statistically significant difference. 
lip, one case of sirenomelia, and one case of hypoplastic left heart in a fetus with no ultrasound before intrauterine fetal death. Within the 31 pregnancies where ultrasound data were available, ultrasound detected 29 pregnancies with associated anomalies (93.5\%). Anomalies that were missed by ultrasound included one case of trisomy 18 and one case of cleft lip/palate. In the control group, seven of the nine anomalies were detected by ultrasound. Those missed included 92,XXYY/46,XY and one case of hypospadias.

\section{DISCUSSION}

In our sample of pathology specimens, fetuses with SUA had a significantly increased incidence of chromosomal $(10.3 \%$ [10/97] vs. $1.0 \%$ [1/97] in controls; $P<0.025)$ and total congenital anomalies $(37.1 \%$ [36/97] vs. 9.3\% [8/97] in controls; $P$ $<0.005)$. The high incidence of congenital anomalies in the controls is reflective of the high-risk population referred to our institution. It is of interest that even with this high background rate, the increase in anomalies in fetuses with SUA is observable. Although the hospital policy only indicates that placentas with multiple births, prematurity, or suspected placental abnormalities should be sent to pathology, it is possible that placentas of infants with birth defects or complications are more likely sent to the pathology department, increasing the incidence of anomalies observed. In order to avoid this potential source of ascertainment bias, controls were selected from the pathology records at this institution. The incidence of SUA in the population at Magee-Women's Hospital was 2.0\% among the placentas studied, which is at the high end of the range reported for unselected newborns, suggesting that the SUA placentas had only a slightly increased chance of being sent to pathology.

Maternal age seemed to have no effect on the incidence of anomalies in SUA cases. Women over 35 years of age are known to be at higher risk for having a child with a chromosomal condition. However, it is important to note that of the 10 cases observed to have a chromosomal condition only $2(20 \%)$ were over 35 years of age and some of these chromosomal anomalies (Table 1) are not related to advanced maternal age. The same average maternal ages (29) and proportions of mothers over 35 (28.6\%) were noted in the case and control groups, indicating that advanced maternal age and associated chromosomal conditions are not a major contributor to the incidence of SUA and related anomalies among our referral population.

The incidence of SGA infants was significantly increased among the SUA cases compared to controls (Table 2), but the pathogenesis of the growth failure is not clear. Although the incidence of SGA infants in cases is more than double that in controls, it seems unlikely that the lack of a second artery interferes with circulation sufficiently to cause these infants to be SGA because the incidence of LGA infants is similar in both groups. The rate of malformations among SGA cases is identical to that in the entire SUA group (both 37\%), suggesting that most SGA cases cannot be explained by anomalies that impair growth. In some SUA fetuses, however, there may be a specific condition, such as a chromosomal abnormality or defective placental circulation that impairs growth and causes SUA.

There was an increased incidence of twinning and maternal nongestational diabetes in our study population. The associations of twinning and maternal diabetes with SUA cannot be explained by malformation syndromes because the incidence of other malformations in the twins and offspring of diabetic mothers is actually less than among the other SUA cases. Because there is no indication that ethnicity or socioeconomic status influences the incidence of SUA, these variables were not considered.

Although our cases were ascertained through pathology, ultrasound information was available for $62(63.9 \%)$ cases and $78(80.4 \%)$ controls. There were no false-positive diagnoses of SUA. False-negatives were frequent with SUA being missed prenatally in 24/62 (38.8\%) of those having ultrasound. An ultrasound-based study in our institution would have found a higher rate of complex malformations and worse prognosis for fetuses diagnosed with SUA because additional anomalies were present in $28 / 38(73.7 \%)$ cases with ultrasound documented SUA, 2/24 (8.3\%) cases in which SUA was missed on ultrasound, and 6/35 (17\%) cases with no ultrasound. This indicates that pathology-based studies such as ours are still useful to ascertain the full range of outcomes associated with SUA, especially at gestational ages when ultrasound may be less reliable in diagnosing SUA. As ultrasound improves in sensitivity, we believe that our study will be relevant to management of future cases with SUA diagnosed prenatally. The literature includes conflicting recommendations regarding management when SUA is recognized prenatally., ${ }^{1,3,11-16}$ There is general agreement that targeted ultrasound, if not already done, is indicated due to the high incidence of associated anomalies, ${ }^{1,3,4,13,15,16}$ but many believe that if no other anomalies are detected on ultrasound, further testing is unnecessary.

In a 1991 study, Nyberg's group ${ }^{17}$ found that prenatal sonography alone was reliable in detecting any major concurrent anomalies, and recommended no alteration in pregnancy management unless additional anomalies are observed. Parilla and colleagues ${ }^{18}$ found only a single case of congenital heart disease after birth among 50 fetuses with apparently isolated SUA on prenatal ultrasound. Because the other 49 fetuses were clinically normal and the 17 that were karyotyped all had normal chromosomes, they felt that amniocentesis was not indicated. Fetal echocardiography, according to Parilla, would have missed the anomaly and was not recommended. In contrast, Budorick et al. ${ }^{13}$ provide data in support of fetal echocardiography. Cardiac malformations were found in $5 \%$ of fetuses with apparently isolated SUA in their study population $(0.8 \%$ to $1 \%$ baseline risk), as well as $50 \%$ of nonisolated SUA.

Our data support the use of detailed ultrasound whenever SUA is suspected. Because our cases were ascertained from pathology records, the SUA was not always recognized prenatally and our evaluation is necessarily retrospective. Only 62 of our cases had ultrasound data and in 28 of these cases (45\%), another anomaly was detected. Two anomalies (trisomy 18 and cleft lip/palate) were missed early in the second trimester. 
In both cases, the SUA was also undetected until delivery. Nevertheless, these cases illustrate that in high-risk pregnancies, including those with SUA, ultrasound early in the second trimester can miss important findings and follow-up later in the second trimester should be considered. Among the 35 cases without ultrasound data, $5(14 \%)$ had anomalies that could have been detected and altered management (triploidy, sirenomelia, bilateral cleft lip/palate and 2 cases with congenital heart disease), and one case had an anomaly probably not recognizable on ultrasound (apparently balanced translocation). The higher incidence of anomalies in cases with ultrasound is probably due to the referral of women with abnormal ultrasound findings, but the incidence of anomalies in both groups exceeds that in controls from the same referral population and is sufficient to justify detailed ultrasound.

Our data also support the recommendation of echocardiography in any pregnancy where SUA is observed prenatally. The majority of the 36 anomalies ( 22 or $61 \%$ ) in our SUA population involved the heart. In four cases, the cardiac anomaly was isolated and in several others it was the major feature identified prenatally, although other anomalies ascertained after birth led to the diagnosis of a syndrome.

Chromosome anomalies are acknowledged to be frequent in fetuses with SUA and multiple anomalies, ${ }^{1,3,4,15}$ but there is disagreement about karyotyping in cases with isolated SUA. Catanzarite's approach ${ }^{19}$ was slightly different, because fetuses with other anomalies on ultrasound were not excluded, and this group found 10 aneuploid fetuses among 82 with SUA. Although all the aneuploid fetuses had other anomalies and/or were SGA, several of these were not identified until the third trimester. Therefore, they recommended karyotype whenever SUA was seen with intrauterine growth retardation or other anomalies. About the same time, Persutte and Hobbins, ${ }^{1}$ concerned about the high incidence of anomalies in fetuses with SUA and the possibility that associated anomalies might be missed on second trimester ultrasound, recommended an extensive work-up, including targeted sonography, fetal echocardiography, fetal genetic testing, enhanced fetal surveillance, as well as careful physical evaluation after delivery.

Given the 10\% incidence of chromosome abnormalities in our SUA study group, we believe that amniocentesis should be offered to all patients with SUA on prenatal ultrasound. It must be noted, however, that $8 / 10$ chromosome anomalies in our study population were detected on ultrasound, one was apparently balanced and not expected to cause birth defects, and only one previously unsuspected clinically significant chromosome anomaly would have been detected by amniocentesis. This is $1 \%$ of our study group and thus places patients with apparently isolated SUA in a risk group for aneuploidy, similar to mothers in their late 30 s or families with a previously chromosomally abnormal child, suggesting that amniocentesis should be offered. In reality, the SUA, as well as the chromosome anomaly, was missed in our trisomy 18 case and thus amniocentesis was not offered. Rather than making a general recommendation for amniocentesis in all cases with SUA, we believe that such patients should be referred for individualized counseling to discuss the implications of SUA on ultrasound and the options for further investigation.

\section{ACKNOWLEDGMENTS}

Thanks to Marshfield Clinic Research Foundation for providing assistance in the preparation of this manuscript through the services of Graig Eldred and Alice Stargardt.

\section{References}

1. Persutte WH, Hobbins J. Single umbilical artery: A clinical enigma in modern prenatal diagnosis. Ultrasound Obstet Gynecol 1995;6:216-229.

2. Moore KL, Persaud TVN. The placenta and fetal membranes. In: Moore KL, Persaud TVN, editors. The developing human: Clinically oriented embryology, 6th ed. Philadelphia: W.B. Saunders, 1998:129-162.

3. Geipel A, Germer U, Welp T, Schwinger E, Gembruch U. Prenatal diagnosis of single umbilical artery: Determination of the absent side, associated anomalies, Doppler findings and perinatal outcome. Ultrasound Obstet Gynecol 2000;15:114-117.

4. Romero R, Pilu G, Jeanty P, Ghidini A, Hobbins JC, editors. Prenatal diagnosis of congenital anomalies. Norwalk: Appleton \& Lange, 1988.

5. Tanimura T, Ezaki KI. Single umbilical artery found in Japanese embryos. Proc Congenit Anomalies Res Assoc Jpn 1968;8:27.

6. Byrne J, Blanc WA. Malformations and chromosome anomalies in spontaneously aborted fetuses with single umbilical artery. Am J Obstet Gynecol 1985;151:340-342.

7. Bryan EM, Kohler HG. The missing umbilical artery, I: Prospective study based on maternity unit. Arch Dis Child 1974;49:844-852.

8. Thummala MR, Raju TNK, Langenberg P. Isolated single umbilical artery anomaly and the risk for congenital malformations: A meta-analysis. J Ped Surg 1998;33:580586.

9. Heifetz SA. Single umbilical artery: A statistical analysis of 237 autopsy cases and review of the literature. Perspect Pediatr Pathol 1984;8:345-378.

10. Usher R, McLean F. Intrauterine growth of live-born Caucasian infants at sea level: standards obtained from measurements in 7 dimensions of infants born between 25 and 44 weeks of gestation. J Pediatr 1969;74:901-910.

11. Bourke WG, Clarke TA, Mathews TG, O'Halpin D, Donoghue VB. Isolated single umbilical artery: The case for routine renal screening. Arch Dis Child 1993;68:600 601.

12. Pavlopoulos PM, Konstantinidou AE, Agapitos E, Christodoulou CN, Davaris P. Association of single umbilical artery with congenital malformations of vascular etiology. Pediatr Dev Pathol 1998;1:487-493.

13. Budorick NE, Kelly TF, Dunn JA, Scioscia AL. The single umbilical artery in a high-risk patient population: what should be offered? J Ultrasound Med 2001;20: 619-627.

14. Wu MH, Chang FM, Shen MR, Yao BL, Chang CH, Yu CH, Hsu CC, Huang KE. Prenatal sonographic diagnosis of single umbilical artery. J Clin Ultrasound 1997;25: 425-430.

15. Rinehart BK, Terrone DA, Taylor CW, Isler CM, Larmon JE, Roberts WE. Single umbilical artery is associated with an increased incidence of structural and chromosomal anomalies and growth restriction. Am J Perinatol 2000;17:229-232.

16. Abuhamad AZ, Shaffer W, Mari G, Copel JA, Hobbins JC, Evans AT. Single umbilical artery: Does it matter which artery is missing? Am J Obstet Gynecol 1995;173: $728-732$.

17. Nyberg DA, Mahony BS, Luthy D, Kapur R. Single umbilical artery: Prenatal detection of concurrent anomalies. J Ultrasound Med 1991;10:247-253.

18. Parilla BV, Tamura RK, MacGregor SN, Geibel LJ, Sabbagha RE. The clinical significance of a single umbilical artery as an isolated finding on prenatal ultrasound. Obstet Gynecol 1995;85:570-572.

19. Catanzarite VA, Hendricks SK, Maida C, Westbrook C, Cousins L, Schrimmer D. Prenatal diagnosis of the two-vessel cord: Implications for patient counseling and obstetric management. Ultrasound Obstet Gynecol 1995;5:98-105. 